\title{
Ainsi en tête de phrase + inversion : une analyse de corpus
}

\author{
Karssenberg, Lena \& Lahousse, Karen \\ Katholieke Universiteit Leuven \\ Lena.Karssenberg@kuleuven.be, Karen.Lahousse@arts.kuleuven.be
}

\section{Introduction}

L'adverbe ainsi est l'un des seuls adverbes qui peut se combiner non seulement avec l'ordre des mots canonique sujet-verbe, mais aussi avec les trois types d'inversion, notamment l'inversion nominale (1a), l'inversion complexe (1b) et l'inversion pronominale (1c).

a. Ainsi + inversion nominale

Le 23 mai 1992, Giovanni Falcone, en poste à Rome où il chapeaute un groupe de magistrats anti-mafia, s'envole pour Palerme à bord d'un avion des services secrets italiens. (...) A 17 heures 56, le véhicule est pulvérisé par une bombe sur l'autoroute qui mène à Palerme. Le juge, sa femme et trois policiers qui l'escortaient trouvent la mort. Ainsi commence le documentaire qui reconstitue minutieusement l'enquête des autorités italiennes. (M426)

b. Ainsi + inversion complexe

Plusieurs commentateurs, professionnels ou occasionnels, déplorent cependant que perdure en Belgique cette culture de l'irresponsabilité généralisée qui permet aux responsables politiques de demeurer à leur poste quelles que soient les circonstances, comme naguère après le drame du stade du Heysel. Ainsi le dessinateur Philippe Geluck, le père du chat obèse à la philosophie paradoxale, dénonce-t-il dans La Libre Belgique le fait que le rapport n'établit pas clairement les responsabilités politiques. (M378)

c. Ainsi + inversion pronominale

Ses calembours suintent l'antisémitisme et il ne rate jamais une allusion au génocide juif pour en relativiser l'ampleur. Ainsi déclarait-il, le 20 novembre 1996, qu'" en France, en 1943, on ne traitait pas les juifs comme on traite aujourd'hui les gens du Front national ». (M269)

Bien que les études consacrées à cet adverbe (Buchmüller 1975, Flament-Boistrancourt 1999, Hybertie 1996, Lahousse 2011, Le Bidois 1952, Molinier 2012 inter alia) soulignent toutes la variété d'interprétations qu'il peut avoir, il existe peu de consensus sur les interprétations avec lesquelles chacun de ces types d'inversion peut se combiner. En outre, la majorité de ces études se fondent sur quelques phrases exemples, et pas sur une analyse de corpus systématique.

Dans cet article, nous présentons les résultats de notre analyse de 171 phrases à ainsi initial qui proviennent de textes journalistiques (Le Monde, indiqué par $M$ dans ce qui suit). Le but principal de cette étude est de déterminer avec quelles interprétations d'ainsi les trois types d'inversion se construisent.

Nous montrerons qu'il existe de claires tendances en ce qui concerne la combinaison 'interprétation d'ainsi + type d'inversion', qui confirment en partie les hypothèses formulées dans les études antérieures, mais qui permettent aussi d'en falsifier quelques-unes. Ainsi, les deux observations les plus intéressantes qui ressortent de notre analyse, et qui contredisent des hypothèses existantes, sont que (i) l'inversion nominale se combine assez souvent avec ainsi de conséquence, et que (ii) l'interprétation «illustrative » 
d'ainsi, qui n'est pas souvent mentionnée dans la littérature, est de loin l'interprétation la plus fréquente quand ainsi se combine avec l'inversion complexe.

La première section de cet article (section 2) présente un très bref survol des principaux travaux sur ainsi et les trois types d'inversion. Dans la section 3, nous présentons une nouvelle classification des interprétations possibles de l'adverbe ainsi, ainsi que plusieurs tests de reformulation pour distinguer entre ces interprétations. Dans la section 4 est présentée notre méthodologie, et l'analyse quantitative et qualitative.

\section{2 État de la question}

Dans la section 2.1, nous présentons les principales significations d'ainsi, indépendamment de l'ordre des mots dans la phrase que cet adverbe introduit. Ensuite, nous présentons brièvement les hypothèses antérieures concernant les différentes interprétations qu'ainsi peut avoir en combinaison avec ces types d'inversion (2.2).

\subsection{Interprétations}

Tous les auteurs qui étudient ainsi (notamment, Blanche-Benveniste 2006, Buchmüller 1975, FlamentBoistrancourt 1999, Guimier 1997, Hybertie 1996, Jonare 1976, Lahousse 2011, Le Bidois 1952, Molinier 2012 et Zenone 1982) sont d'accord pour dire qu'ainsi peut fonctionner comme adverbe de manière et comme adverbe de phrase.

Ainsi de manière (2) se paraphrase par de cette manière et renvoie à une manière spécifiée dans le contexte gauche de la phrase qu'il introduit.

(2) Ainsi de manière

a. Sous la Restauration, beaucoup d'orateurs préparaient leur discours à l'avance en faisant de véritables ouvrages qui étaient imprimés sous forme de petits factums. Ainsi travaillait RoyerCollard. (Molinier 2012 : 122)

b. Il écrivait avec une sorte de distraction concentrée, comme on crayonne sur le bloc du téléphone : on écoute de moins en moins et c'est le dessin qui s'impose. Ainsi écrivait Alexandre, se réfugiant dans les pleins et les déliés de cette écriture sage, de ce crayonnement appliqué. (Pennac ; Lahousse 2011 : 94)

Ainsi quotatif (3) a comme fonction de marquer ce qui précède comme discours direct.

(3) Ainsi quotatif

a. Ainsi dit le renard. (Flament-Boistrancourt 1999: 149, le contexte manque)

b. Ainsi pensait Jeanne. Ainsi chuchota-t-elle. Ainsi raisonnait Mary-Anne. Ainsi parlait Zarathustra. (Hybertie 1996 : 47, le contexte manque)

Quand ainsi fonctionne comme un adverbe de phrase, il peut avoir plusieurs interprétations ${ }^{1}$, dont la conséquence (4) et l'illustration (5). Dans (4), la phrase introduite par ainsi est la conséquence du contexte gauche ; ainsi crée ou explicite donc un lien de cause-conséquence.

(4) Ainsi de conséquence

Je n'ai pas proposé à M. de Narbonne de dîner ici. Ainsi, tu es le maître de dire ce qui te convient. (Hybertie $1996: 48$ )

Dans (5), par contre, la phrase introduite par ainsi n'est pas une conséquence mais un exemple de la phrase précédente : le fait que les hommes chassent et les femmes gardent la caverne et les enfants est présenté comme une illustration de la répartition bien définie des rôles mentionnée dans le contexte gauche. 
(5) Ainsi illustratif

À l'époque de ces sociétés primitives, les rôles de chaque sexe étaient clairement définis, et chacun savait ce qu'il avait à faire. Ainsi les hommes chassaient et les femmes gardaient la caverne et les enfants. (Molinier $2012: 125$ )

Aux interprétations de conséquence et d'illustration, Molinier (2012) ajoute la précision (6a) et la conclusion (6b).

(6) ainsi de précision

Le site propose assez souvent des ventes de la collection Freeman Porter avec des réductions pouvant aller jusqu'à 70\%. Ainsi, il est possible d'acheter un jean de la marque à moindre frais (20 euros par exemple).

b. Ainsi conclusif

À l'époque de ces sociétés primitives, les hommes chassaient et les femmes gardaient la caverne et les enfants. Ainsi, les rôles de chaque sexe étaient clairement définis, et chacun savait ce qu'il avait à faire. (Molinier $2012: 125$ )

Bien que plusieurs interprétations d'ainsi aient donc été mentionnées, il n'existe pas de critères clairement définis pour les distinguer. Dans la section 3, nous présenterons de tels critères qui seront ensuite appliqués à notre corpus (section 4). Mais nous traitons d'abord de la question des types d'inversion dans la section suivante.

\subsection{Ainsi + inversion}

L'inversion nominale a été étudiée par, entre beaucoup d'autres, Cornish (2001), Fournier (1997), Lahousse (2011) et Marandin (1999). Selon plusieurs auteurs, l'inversion nominale se combine normalement avec ainsi de manière (Le Bidois 1952, Lahousse 2011) (cf. (4)). Molinier (2012) affirme même que l'inversion nominale impose une lecture d'ainsi comme adverbe de manière, comme dans l'exemple $(7)^{2}$.

(7) Ainsi de manière + inversion nominale

Ainsi travaillait Paul. (Molinier 2012 : 126)

L'inversion nominale se combine également avec ainsi quotatif (cf. (3)). Flament-Boistrancourt (1999) présente aussi l'exemple (iia) pour montrer qu'ainsi illustratif se combine avec l'inversion nominale. Il est à noter, cependant, que dans la classification de Lahousse (2011), (8a) est un cas d' "inversion focus », qui s'explique ici par le caractère lourd du sujet; notons que cet exemple serait également acceptable sans ainsi $(8 \mathrm{~b})$.

(8) Ainsi illustratif + inversion nominale

a. Une autorisation spéciale d'expédition n'est pas nécessaire pour toutes les marchandises. Ainsi, peuvent être envoyées sans autorisation les marchandises suivantes... (FlamentBoistrancourt 1999 : 149)

b. Peuvent être envoyées sans autorisation les marchandises suivantes...

Selon Le Bidois (1952), Buchmüller (1975) et Lahousse (2011), l'inversion complexe se combinerait surtout avec ainsi adverbe de phrase (cf. 4). Molinier (2013) affirme même que ce type d'inversion induit nécessairement une interprétation d'ainsi comme adverbe de phrase (9).

(9) Ainsi adverbe de phrase + inversion complexe

Ainsi, Pierre travaillait-il. (Molinier 2012 : 122; le contexte manque) 
Riegel, Pellat \& Rioul (2011 : 256) et Lahousse (2011) affirment qu'ainsi ne peut pas être quotatif quand il se combine avec l'inversion complexe.

Selon la littérature, l'inversion pronominale se combine avec ainsi de manière (10a), ainsi quotatif (10b), ainsi de conséquence (10c) et ainsi illustratif (10d).

(10) a. Ainsi de manière + inversion pronominale

Riche, heureux, adulé à son tour, jouissant de toutes les voluptés, gourmand, débauché, ainsi vivait-il à Venise, honoré de tous et ayant Le Titien pour ami intime. (J. Richepin, dans Molinier $2012: 123)$

b. Ainsi quotatif + inversion pronominale

"Nous avons un staff de quatre entraîneurs qui vont diriger les Lions Indomptables de football ». Ainsi parlait-il, hier, au sujet de la fédération du quartier Tsinga. (www, dans Molinier $2012:$ 123)

c. Ainsi de conséquence + inversion pronominale

Nous avons loué une maison de compagne. Ainsi pourrons-nous passer des week-ends au grand air. (Flament-Boistrancourt 1999 : 147)

d. Ainsi illustratif + inversion pronominale

Je ne raconterai pas les menues tribulations auxquelles ma décision donna naissance. Ainsi ai-je dû faire commencer des travaux dans mon appartement. (Basé sur Romilly, p.247 dans Guimier $1997: 85)$

Il semble donc que l'inversion pronominale est moins contrainte que les autres types d'inversion en ce qui concerne les interprétations d'ainsi avec lesquelles elle peut se combiner.

Pour résumer, les trois combinaisons suivantes sont décrites dans les travaux antérieurs sur ainsi :

- ainsi de manière / quotatif + inversion nominale

- ainsi adverbe de phrase + inversion complexe

- $\quad$ tous les ainsi + inversion pronominale

Dans la section 5 nous vérifierons si ces trois combinaisons rendent bien compte de la réalité linguistique telle qu'elle est observée dans un corpus. Avant de faire cela, nous devons formuler des critères clairs permettant de distinguer les différentes interprétations d'ainsi. C'est à cela qu'est consacrée la prochaine section 3 .

\section{Les cinq interprétations}

Dans les prochaines sous-sections, passeront en revue les cinq significations qu'ainsi initial peut avoir selon nous : ainsi de manière et ainsi quotatif (3.1), et ainsi introduisant une conséquence, une précision et une illustration (3.2). Dans la section 3.3 sera présenté un bilan des critères distinctifs des interprétations.

\subsection{Ainsi de manière \& ainsi quotatif ${ }^{3}$}

Ainsi adverbe de manière (11a) se distingue des autres interprétations par la possibilité d'être paraphrasé par de cette manière (11b) et par le fait que son antécédent peut être récupéré par l'interrogatif de quelle manière (11c). 
(11) Ainsi de manière

a. Le 21 janvier 1995, l'ETA abattait, en plein centre de Saint-Sébastien, le dirigeant du PP au Pays basque et adjoint au maire de cette ville. Ainsi commençait la série macabre [de meurtres]. (M550)

b. De cette manière commençait la série.

c. De quelle manière commençait la série?

Dans les cas de ce type, ainsi renvoie à la façon dont se déroule l'événement dénoté par le verbe (la façon de commencer), et fonctionne donc comme un adverbe de manière verbal.

Ainsi quotatif ne désigne pas une manière, mais sert à signaler que le contexte précédent relève du discours direct (12a). Ce type d'ainsi ne se paraphrase pas par de cette manière (12b) et son antécédent peut être récupéré par une question introduite par qu'est-ce que ou que (12c) plutôt que par de quelle manière (12d).

(12) Ainsi quotatif

a. "Il ne cherche plus qu'à animer le peuple contre le souverain pontife pour qu'on se révolte contre lui et ses ministres, (...). » Ainsi dit Machiavel d'un dominicain condamné au bûcher, ainsi beaucoup ont pensé et écrit de Raymond Léopold Bruckberger. (M237)

b. * De cette manière dit Machiavel.

c. Qu'est-ce que Machiavel dit? Que dit Machiavel?

d. * De quelle manière dit Machiavel?

\subsection{Ainsi de conséquence, ainsi de précision \& ainsi illustratif}

En tant qu'adverbe de phrase, ainsi peut introduire une conséquence, une précision ou une illustration.

Ainsi de conséquence explicite un lien de cause-conséquence entre le contexte gauche et la phrase qu'il introduit. Dans (13a), par exemple, le fait que le jugement de 1894 a été annulé est la cause du fait que Dreyfus sera ramené en France. Dans cet exemple, ainsi de conséquence ne se paraphrase pas par de cette manière (13b), mais par par conséquent (13c). La paraphrase de quelle manière? n'est pas acceptable non plus (13d).

(13) Ainsi de conséquence - Type 1

a. [Zola] apprendra le suicide d'Henry, (...) puis la décision de la Cour de cassation, qui déclarera recevable, le 27 octobre, la requête en révision déposée par Lucie Dreyfus et ordonnera un supplément d'instruction. Oui, cette fois-ci la Vérité est en marche... Zola est averti, le 3 juin 1899, de l'annulation du jugement de 1894 qui condamnait Dreyfus. Ainsi Dreyfus va-t-il être ramené en France et à nouveau jugé. (M177)

b. * De cette manière Dreyfus va(-t-il) être ramené en France.

c. Par conséquent, Dreyfus va être ramené en France.

d. * De quelle manière Dreyfus va-t-il être ramené en France?

Cependant, le corpus contient également des cas où ainsi introduit une conséquence (14a) et se laisse paraphraser soit par de cette manière (14b), soit par par conséquent (14c), la paraphrase de quelle manière? n'étant pas exlcue (14d).

(14) Ainsi de consequence - Type 2

a. Il n'est donc pas exclu que les taux d'intérêt soient prochainement revus à la baisse par la Reserve Bank, de façon à accompagner une croissance plus soutenue. 


\begin{abstract}
majeure. (M569)
b. De cette manière le PIB pourrait s'accroître...

c. Par conséquent le PIB pourrait s'accroître...

d. De quelle manière le PIB pourrait-il s'accroître?
\end{abstract}

Ainsi le PIB pourrait-il s'accroître d'un peu plus de 2\% cette année, sans risque de dérive

La possibilité de reformuler ainsi soit comme de cette manière, soit comme par conséquent n'a pas encore été remarquée dans la littérature sur ainsi. Pour expliquer ces cas, nous proposons de distinguer entre deux types de conséquence : la conséquence voulue (14) et la conséquence non intentionnelle (13). Quand ainsi introduit la conséquence voulue, il y a un agent qui a l'intention d'obtenir un certain résultat en faisant quelque chose. Dans (14), par exemple, la Reserve Bank (l'agent) a l'intention de faire croître le PIB, en baissant les taux d'intérêt. Ainsi renvoie donc à une manière qui permet d'obtenir un certain résultat, c'est-à-dire une conséquence voulue. Dans (13), par contre, l'annulation du jugement de 1894 a comme conséquence que Dreyfus va être ramené en France, mais la phrase ne contient pas d'agent, ce qui est, selon nous, en corrélation avec le fait qu' ainsi ne peut pas être remplacé par de cette manière. Remarquons toutefois que ainsi et la paraphrase de quelle manière dans (14) ne renvoient pas à la manière dont se déroule l'événement dénoté par le groupe verbal, comme c'est le cas en (11). Dans (14), ainsi et de quelle manière expriment plutôt une condition : Le PIB pourraît s'accroître si la Reserve Bank fait ceci : baisser les taux d'intérêt.

Selon nous, la distinction entre la conséquence voulue et la conséquence non intentionnelle pourrait être le résultat du parcours diachronique d'ainsi, et plus en particulier de l'évolution de son interprétation. En effet, à travers les langues, les déictiques de manière se grammaticalisent souvent pour devenir des marqueurs de conséquence (König 2012), et plus généralement, Traugott (1997) affirme que les adverbes intégrés à la proposition (comme le sont les adverbes de manière verbale) se grammaticalisent souvent en des adverbes de phrase, devenant finalement des marqueurs de discours (voir aussi Lamiroy \& Charolles 2004, Lamiroy 2005 et Charolles \& Lamiroy 2008 pour une analyse similaire des adverbes seulement, simplement et heureusement). Or, notre hypothèse est qu'au début, ainsi ne désigne que la manière, et qu'ensuite, l'adverbe commence à exprimer la conséquence voulue, qui combine la manière et la conséquence. Après cela, dans certains contextes, ainsi perd sa valeur de manière pour ne garder que l'interprétation de conséquence (= non intentionnelle). Dans cette optique, ainsi de conséquence voulue pourrait être considéré comme un 'bridging context' (Heine 2002, Evans \& Wilkins 2000), un contexte de transition entre ainsi de manière et ainsi de conséquence non intentionnelle. De plus, l'analyse de Lamiroy \& Charolles (2004), Lamiroy (2005) et Charolles \& Lamiroy (2008) renforce notre hypothèse du processus de grammaticalisation : selon ces auteurs (i) la position d'un adverbe en tête de phrase ainsi que (ii) son sens fondamentalement 'relationnel' favorisent un processus de grammaticalisation. Ce deuxième point est essentiel selon ces auteurs, parce que c'est le sens relationnel de l'adverbe qui permettrait son emploi comme un connecteur. Or, il est clair qu'ainsi en tant qu'adverbe de manière (i) peut apparaître en position initiale de la phrase et (ii), renvoyant à un antécédent dans le contexte précédent, ainsi a nécessairement une valeur relationnelle. ${ }^{4}$

$\mathrm{Vu}$ le nombre limité d'occurrences d'ainsi de conséquence, que ce soit la conséquence voulue ou non intentionnelle, nous ne tiendrons pas compte de cette distinction dans notre analyse quantitative. Quand un exemple d'ainsi se laisse paraphraser par de cette manière et par conséquent (= conséquence voulue), nous la mettrons dans la catégorie 'conséquence'.

Revenons maintenant à notre classification des différentes interprétations. A part la conséquence, ainsi peut aussi introduire une précision ou une explicitation de quelque chose qui se trouve dans le contexte gauche. Dans (15a), par exemple, le fait que le ministère n'élude pas la question de l'ennui est élaboré par la précision des questions qui traitent de l'ennui (15b). Ainsi de précision ne se remplace pas par de cette manière (15c), ni par par conséquent (15d). 
(15) Ainsi de précision

a. L'ennui. La question est aussi vieille que l'école, et le questionnaire du ministère de l'éducation ne l'élude pas. Ainsi est-il demandé aux lycéens ce qui leur apparaît "inutile et sans intérêt 》, mais aussi ce qui les "ennuie» dans ce qu'ils jugent pourtant "important" d'apprendre. (M478)

b. Pour être précis, on demande aux lycéens...

c. * De cette manière, on demande aux lycéens...

d. * Par conséquent, on demande aux lycéens...

Ainsi illustratif introduit un exemple d'une proposition ou d'une classe plus générale qui se trouve dans le contexte. Dans (16a), par exemple, Philippe Geluck fait partie des plusieurs commentateurs. Dans cet emploi, ainsi ne commute ni avec de cette manière (16b) ni avec par conséquent (16c), mais se laisse bien remplacer par par exemple 5 (16d). Le lecteur pourra facilement vérifier que cela ne vaut pas pour les autres interprétations.

(16) Ainsi illustratif

a. Plusieurs commentateurs, professionnels ou occasionnels, déplorent cependant que perdure en Belgique cette culture de l'irresponsabilité généralisée qui permet aux responsables politiques de demeurer à leur poste quelles que soient les circonstances, comme naguère après le drame du stade du Heysel. Ainsi le dessinateur Philippe Geluck, le père du chat obèse à la philosophie paradoxale, dénonce-t-il dans La Libre Belgique le fait que le rapport n'établit pas clairement les responsabilités politiques. (M378)

b. * De cette manière, Philippe Geluck dénonce le fait que...

d. * Par conséquent, Philippe Geluck dénonce le fait que...

d. Philippe Geluck, par exemple, dénonce le fait que...

\subsection{Bilan}

Le tableau 1 présente un bilan des critères qui permettent de distinguer entre les différentes interprétations d'ainsi.

\begin{tabular}{|c|c|c|c|c|c|}
\hline & Manière & Quotatif & Conséquence & Précision & Illustration \\
\hline $\begin{array}{l}\text { Antécédent récupéré par } \\
\text { 'de quelle manière...?' }\end{array}$ & + & - & $-1+$ & - & - \\
\hline $\begin{array}{l}\text { Antécédent récupéré par } \\
\text { 'qu'est-ce que...'? }\end{array}$ & - & + & - & - & - \\
\hline Paraphrase 'de cette manière' & + & - & $-1+^{6}$ & - & - \\
\hline Paraphrase 'par conséquent' & - & - & + & - & - \\
\hline Paraphrase 'pour être précis' & - & - & - & + & - \\
\hline Paraphrase 'par exemple' & - & - & - & - & + \\
\hline
\end{tabular}

Tableau 1 - Critères différentiels des interprétations

La prochaine section présente les résultats de l'application de ces tests à chacun des exemples d'inversion qui se trouvent dans notre corpus. 


\section{Analyse}

Avant de présenter les résultats quantitatifs (section 4.2) et l'analyse qualitative des exemples nonprototypiques dans la section 4.3 , nous présenterons brièvement le corpus que nous avons utilisé dans la prochaine section.

\subsection{Le corpus Le Monde (1998)}

Cette étude est basée sur le corpus Le Monde (1998). La commande de recherche de tous les contextes où ainsi est immédiatement précédé d'un point final [. Ainsi] a résulté en 633 exemples, qui ont été soumis à un tri manuel visant à sélectionner les cas où ainsi introduit une phrase verbale ${ }^{7}$. Dans 366 phrases $(68,1 \%)$, ainsi est suivi de l'ordre des mots canonique sujet verbe (qui ne seront pas considérées dans cet article), alors que dans 171 exemples $(31,8 \%)$, ainsi introduit une phrase à inversion. La répartition des types d'inversion est présentée dans le tableau 2 :

\begin{tabular}{|l|l|l|}
\hline Type d'inversion & $\mathrm{N}$ & $\%$ \\
\hline Inversion complexe & 73 & $42.4 \%$ \\
\hline Inversion nominale & 59 & $34.9 \%$ \\
\hline Inversion pronominale & 39 & $22.7 \%$ \\
\hline Total & 171 & $100 \%$ \\
\hline
\end{tabular}

Tableau $2-$ Ainsi + inversion

\subsection{Résultats quantitatifs}

Le tableau 3 présente les résultats quantitatifs de l'application au corpus des tests présentés dans la section 3 .

\begin{tabular}{|l|c|c|c|c|c|c|}
\hline & \multicolumn{2}{|c|}{ Inversion nominale } & \multicolumn{2}{c|}{ Inversion complexe } & \multicolumn{2}{c|}{ Inversion pronominale } \\
\hline & $\mathrm{N}$ & $\%$ & $\mathrm{~N}$ & $\%$ & $\mathrm{~N}$ & $\%$ \\
\hline Manière & $\mathbf{4 2}$ & $\mathbf{7 1 . 2 \%}$ & 6 & $8.2 \%$ & 5 & $12,8 \%$ \\
\hline Quotatif & 3 & $5.1 \%$ & 0 & $0 \%$ & 1 & $2,6 \%$ \\
\hline $\begin{array}{l}\text { Conséquence (voulue/ } \\
\text { non intentionelle) }\end{array}$ & 19 & $20.3 \%$ & 16 & $21.9 \%$ & $\mathbf{1 5}$ & $\mathbf{3 8 , 5 \%}$ \\
\hline Précision & 0 & $0 \%$ & 6 & $8.2 \%$ & 8 & $20.5 \%$ \\
\hline Illustration & 2 & $3.4 \%$ & $\mathbf{4 5}$ & $\mathbf{6 1 . 6 \%}$ & 10 & $25.6 \%$ \\
\hline Total & 59 & $100 \%$ & 73 & $100 \%$ & 39 & $100 \%$ \\
\hline
\end{tabular}

Tableau 3 - Classification des interprétations selon le type d'inversion

Ces données confirment deux observations antérieures (voir section 2) : (i) l'interprétation de manière est la plus fréquente quand ainsi précède l'inversion nominale ( $71.2 \%$ des cas d'inversion nominale). (ii) Quand ainsi introduit une phrase à inversion complexe ou pronominale, par contre, ainsi est 
majoritairement un adverbe de phrase (91.8\% des cas d'inversion complexe, $84,6 \%$ des cas d'inversion pronominale). Ces co-occurrences d'interprétations + type d'inversion sont illustrées dans (17):

(17) a. Ainsi de manière + inversion nominale

Comme au cirque, dans le public un homme éclate de rire. Le président Castagnède ne bronche pas. Ainsi va la fin du procès de Maurice Papon, soumis à des rumeurs que le juge au cours des premiers mois s'empressait de réprimander promptement. (M389)

b. Ainsi illustratif + inversion complexe

Apparemment, la médiatisation qui a entouré la sortie du film Lucie Aubrac, de Claude Berri, a heurté certains résistants. Ainsi André Laroche, soixante-treize ans, du Centre de la résistance et de la déportation de Lyon, proche de Gérard Chauvy, s'insurge-t-il contre un film « qui tend à faire croire qu'il y avait des grands et des petits résistants, alors qu'il n'y avait que des résistants ». (M418)

c. Ainsi de conséquence + inversion pronominale

Professionnels et élus doivent aussi intégrer les nouveaux comportements des touristes, avec des séjours de plus en plus courts. Mais, surtout, remarque Guy Julien, les demandes sont désormais souvent tournées vers "la notion de terroir, de pays et d'identité ». Ainsi souhaite-t-il que les stations s'intègrent mieux à leur environnement, pour ne plus être "considérées par les gens comme un outil extérieur gérant une migration qui ne les regarde pas ». (M323)

Cependant, nos données permettent aussi d'invalider certaines observations antérieures. Premièrement, contrairement à ce qui est suggéré par les travaux antérieurs sur ainsi + inversion, l'inversion nominale se combine aussi avec ainsi de conséquence dans un nombre non négligeable de cas, à savoir environ $20 \%$. L'idée que l'inversion nominale impose une lecture d'ainsi comme adverbe de manière (Molinier 2012) ne vaut donc pas dans notre corpus. Deuxièmement, l'inversion complexe s'avère se combiner avec ainsi de manière à plusieurs reprises, ce qui n'était pas non plus prédit par la littérature. Dernièrement, l'interprétation illustrative, qui n'est pas étudiée de façon détaillée dans les autres travaux sur ainsi, est de loin l'interprétation la plus fréquente quand ainsi précède l'inversion complexe.

Notre corpus contient quelques exemples qui ne s'inscrivent pas dans les tendances générales : il s'agit des phrases où (i) l'inversion nominale est précédée par ainsi de conséquence ou ainsi illustratif et (ii) des phrases où ainsi de manière précède l'inversion complexe ou pronominale. Ces cas, que nous pouvons qualifier comme non-prototypiques, seront analysés dans la prochaine section.

\subsection{Cas non-prototypiques}

Dans ce qui suit nous expliquerons les cas qui vont à l'encontre des tendances générales dans notre corpus.

\subsubsection{Ainsi de conséquence / illustratif + inversion nominale}

Comme nous venons de voir, l'inversion nominale se retrouve de façon inattendue après ainsi de conséquence dans environ 20\% des cas, ce qui n'était pas prédit par Molinier (2012). Comment ces cas peuvent-ils être expliqués?

Selon Lahousse (2011), l'inversion nominale doit toujours être justifiée par la présence d'un topique ou d'un focus en position initiale de phrase. Or, dans la mesure où la cause peut être la cause immédiate, elle peut fonctionner comme le topique spatio-temporel, comme dans l'exemple (18a). Dans cet exemple, sa fonction en tant que cadre spatio-temporel devient clair par le fait que l'ajout d'une deuxième spécification temporelle est douteuse (18b). 
(18) Ainsi de conséquence + inversion nominale

a. De 1935 à 1939, il est responsable du secteur classique du Théâtre de Rouen. Il y rencontrera la comédienne Jacqueline Morane, avec laquelle il aura trois enfants. En septembre 1940, Louis Jouvet lui confie la partie de sa troupe qui ne partira pas en Amérique du Sud. Ainsi naît la coopérative La Comédie en Provence. (M159)

b. ???... Ainsi naît à ce moment la coopérative.

Etant un topique spatio-temporel, ainsi justifie alors l'inversion nominale. Ceci est le cas dans la majorité des exemples de 'ainsi de conséquence + inversion'. Remarque lecteur : peut-on dire que ce sont tous les cas où ainsi a vraiment une valeur anaphorique qui permettent l'inversion nominale ? cf aussi ainsi de manière. D'autre part, l'hypothèse de Lahousse (2011) nous permet également d'expliquer pourquoi il y a si peu de cas de 'ainsi illustratif + inversion nominale'. Ainsi illustratif n'a pas de valeur référentielle : contrairement à ainsi de conséquence, il ne renvoie pas à une situation concrète, mais marque uniquement le lien entre le contexte et l'exemple qu'il introduit. Ainsi illustratif ne peut donc pas non plus fonctionner comme topique ou focus. Ceci explique pourquoi l'inversion nominale ne se combine que rarement avec ainsi illustratif. Dans les deux cas où ainsi illustratif introduit quand même une phrase à inversion nominale il doit donc y avoir une autre raison pour laquelle le locuteur a opté pour l'inversion nominale plutôt que pour un autre ordre de mots. En effet, dans (19), le sujet est indéfini (un minuscule vase), et les sujets indéfinis en position initiale tendent à être évités en français. En outre, la phrase à droite commence par une anaphore qui renvoie au référent d'un minuscule vase. L'inversion permet donc de rapprocher antécédent et anaphore, ce qui enlève des ambiguïtés potentielles.

(19) Ainsi illustratif + inversion nominale

Pour jouer sur le registre émotionnel, Volkswagen a entrepris de multiplier les références à la contre-culture des années 60 et 70, qui fit la part belle à sa petite voiture. Ainsi a été installé sur la planche de bord, à main droite juste à côté du volant, un minuscule vase, pas plus gros que le pouce. Cet accessoire surmonté d'une petite bague percée pourra accueillir une fleur, clin d'œil complice au flower power de la fin des années 60. (M409)

Dans (20), l'agriculture sur abattis-brûlis est un exemple des victoires techniques qui repoussent d'abord les limites mais sont ensuite remplacées par autre chose. L'inversion nominale s'y explique par le fait que le sujet est la nouvelle information, qui suit de préférence l'information connue. De plus, l'agriculture sur abattis-brûlis est reprise par l'anaphore cette agriculture dans la phrase suivante, et l'inversion nominale a comme effet positif pour la cohésion du texte que les deux ne sont pas trop éloignés l'une de l'autre.

\section{Ainsi illustratif + inversion nominale}

(...) ̀̀ chaque fois que l'homme remporte une victoire technique qui lui permet de repousser les limites que lui imposent les éléments dont il dispose, tout se passe comme s'il en profitait pour proliférer jusqu'à atteindre de nouvelles limites. Ainsi serait apparue, vers 10000 ans avant notre ère, l'agriculture sur abattis-brûlis, en réponse aux insuffisances de la technique jusqu'alors pratiquée, à savoir la prédation opérée par chasse et cueillette. (...) Mais le déboisement gigantesque opéré par cette agriculture "fut sans doute le plus grand bouleversement écologique de l'histoire », estiment nos auteurs, et il a bien fallu inventer autre chose. (M89)

\subsubsection{Ainsi de manière + inversion complexe/ pronominale}

Nous avons vu dans la section 2.2 que selon les analyses antérieures d'ainsi, l'inversion pronominale est moins contrainte que l'inversion complexe en ce qui concerne les interprétations avec lesquelles ce type d'inversion peut se combiner. Or, il s'avère que dans tous les exemples où ainsi de manière se combine avec l'inversion complexe et pronominale (11 au total), l'inversion nominale est exclue parce que le verbe est suivi d'un objet direct, comme l'illustrent (21a,b) : 
(21) a. Ainsi de manière + inversion complexe

Alors la guerre nous a pris comme une ivresse. Sous une pluie de fleurs, nous étions partis dans une atmosphère d'ivresse de roses et de sang. Ainsi Ernst Jünger décrit-il son expérience du début de la guerre de 1914. (M370)

b. Ainsi de manière + inversion pronominale

Mais la distribution des aires forme une mosaïque d'ensembles interreliés, d'une aire à l'autre, d'un hémisphère à l'autre. Ainsi définit-il la "connectivité réciproque " du cerveau humain (...). (M524)

Cela nous amène à affirmer qu'ainsi de manière se combine par défaut avec l'inversion nominale, sauf quand ce type d'inversion est exclu pour des raisons syntaxiques, auxquels cas le locuteur opte pour un des deux autres types d'inversion. Comme le contexte d'apparition d'ainsi de manière est pareil pour l'inversion complexe et l'inversion pronominale, il nous semble que l'inversion pronominale n'est pas moins contrainte que l'inversion complexe. Cela est renforcé par le fait et que l'inversion pronominale ne se combine avec ainsi quotatif qu'une fois dans notre corpus (22).

(22) Ainsi quotatif + inversion pronominale

Oui, nous avons écrit cela. De Dreyfus : "C'était l'ennemi juif trahissant la France ». De Zola défendant l'innocent : " Etripez-le! » Des juifs : " Contre le Christ qui les a maudits, et dont ils demeurent les ennemis farouches, ils voudraient pouvoir soulever toutes choses: leur haine va jusqu'au délire ». Ainsi écrivait-on, il y a cent ans dans La Croix. (M188)

\section{Conclusion}

Dans cet article nous avons étudié l'adverbe ainsi suivi de trois types d'inversion : inversion nominale, complexe et pronominale. Nous avons proposé une classification de cinq interprétations principales d'ainsi (manière, quotatif, conséquence, précision, illustration). Un intérieur de l'interprétation de la conséquence, nous avons proposé de distinguer entre la conséquence voulue et la conséquence non intentionnelle, ce qui nous a amené à formuler une hypothèse sur le parcours diachronique d'ainsi qui mériterait d'être testé dans un corpus diachronique. Nous avons également souligné qu'il vaudrait la peine de comparer ainsi à ses équivalents dans d'autres langues telles que le néerlandais, l'allemand et l'anglais. Nous avons également proposé des tests de reformulation qui permettent de distinguer les différentes interprétations les unes des autres. En appliquant ces tests à un corpus de 171 occurrences de 'ainsi + inversion', nous avons pu montrer qu'il existe des tendances en ce qui concerne les interprétations avec lesquelles les différent types d'inversion se combinent.

Comme prédit par les travaux antérieurs sur ainsi, (i) l'inversion nominale se combine souvent avec ainsi de manière alors que (ii) l'inversion complexe et l'inversion pronominale sont surtout introduites par ainsi adverbe de phrase. Cependant, nos données ont aussi montré que l'inversion nominale est introduite par ainsi de conséquence dans un nombre non négligeable de cas (environ 20\%). En nous appuyant sur l'analyse de la structure de l'information de l'inversion nominale de Lahousse (2011), nous expliquons les cas où ainsi de conséquence introduit l'inversion nominale par le fait que l'adverbe fonctionne dans ces cas comme un topique spatio-temporel, qui favorise aussi l'inversion nominale dans d'autres contextes d'inversion locative (voir Marandin 2001 et Bonami, Godard, \& Marandin 1999). L'analyse de la structure informationelle permet également d'expliquer la quasi-absence de la combinaison 'ainsi illustratif + inversion nominale' : dans cette interprétation, ainsi n'a pas de valeur référentielle, est incapable d'assumer le rôle de topique ou de focus. Contrairement à l'inversion nominale, par contre, l'inversion complexe s'avère se combiner très fréquemment avec ainsi illustratif, même beaucoup plus souvent qu'avec ainsi de conséquence. Une autre observation que nous avons faite est que l'inversion complexe ainsi que l'inversion pronominale ne se combinent avec ainsi de manière qu'aux cas où l'inversion nominale est exclue en raison de la présence d'un objet direct. Cela nous amène à affirmer 
que, contrairement à ce qu'affirment certaines analyses antérieures d'ainsi + inversion, l'inversion pronominale n'est pas moins contrainte que l'inversion complexe en ce qui concerne les interprétations d'ainsi avec lesquelles ce type d'inversion se combine. La conclusion générale de cet article est donc que le choix du type d'inversion ne dépend pas uniquement de la sémantique d'ainsi; des facteurs syntaxiques entrent également en jeu.

\section{Références bibliographiques}

Bonami, O., Godard, D., \& Marandin, J.-M. (1999). Constituency and Word Order in French Subject Inversion. In G. Bouma, E. . Hinrichs, G.-J. M. Kruiff, \& T. Oehrle (Eds.), Constraints and Resources in Natural Language Syntax and Semantics (pp. 21-40). Stanford: Stanford University Press.

Blanche-Benveniste, C. (2006). Les clivées françaises de type : C'est comme ça que, C'est pour ça que, c'est là que tout a commencé. Moderna Språk, 100(2), 273-287.

Buchmüller, A. (1975). L'Inversion Complexe. Universität Zürich.

Charolles, M. \& Lamiroy, B. (2007). Du lexique à la grammaire : Seulement, simplement, uniquement. Cahiers de Lexicologie 90(1), 93-117.

Lamiroy, B. \& Charolles, M. (2008). Grammaticalisation des adverbes uniquement, seulement et simplement. In Camugli, C., Constant, M. Dister, A. (éds). http://infolingu.univ-mlv.fr/Colloques/Bonifacio/actes.html

Cornish, F. (2001). L'inversion locative en français, italien et anglais: propriétés syntaxiques, sémantiques et discursives. Les cahiers de grammaire, 26, 101-123.

Desmets, M. (2013). Les consécutives et la manière. Scolia, 27, 81-102.

Flament-Boistrancourt, D. (1999). Quelques aspects d'ainsi et aussi consécutifs à la lumière d'un point de vue de nonfrancophone. Le Gré des Langues, 15, 142-180.

Fournier, N. (1997). La place du sujet nominal dans les phrases à complément prépositionnel initial. In C. Fuchs (Ed.), La place du sujet en français contemporain (pp. 97-132). Louvain-la-Neuve: Duculot.

Guimier, C. (1996). Les adverbes du français: le cas des adverbes en -ment. Paris: Ophrys.

Guimier, C. (1997). La place du sujet clitique dans les énoncés avec adverbe initial. In C. Fuchs (Ed.), La place du sujet en français contemporain (pp. 43-96). Louvain-la-Neuve: Duculot.

Heine, B. (2002). On the role of context in grammaticalization. In New reflections on grammaticalization (pp. 83101). Amsterdam/Philadelphia: John Benjamins.

Hybertie, C. (1996). La conséquence en français. Paris: Ophrys.

Jonare, B. (1976). L'inversion dans la principale non-intterogative en français contemporain. Uppsala: Acta Universitatis Upsaliensis.

Karssenberg, L. (2013). Ainsi en tête de phrase: une analyse de corpus. Mémoire de Master. Katholieke Universiteit Leuven.

König, E. (2012). Le rôle des déictiques de manière dans le cadre d'une typologie de la déixis. Bulletin de La Société de Linguistique de Paris, 107(1), 11-42.

Lahousse, K. (2011). Quand passent les cigognes: Le sujet nominal postverbal en français moderne. Paris: Presses Universitaires de Paris. 
Lahousse, K., \& Lamiroy, B. (2014a). C'est ainsi que : grammaticalisation ou lexicalisation ou les deux à la fois ? (manuscrit)

Lahousse, K., \& Lamiroy, B. (2014b). Grammaticalisatie en taalvergelijking: Nederlands het is zo dat vs Frans c'est ainsi que. (manuscrit)

Lamiroy, B., \& Charolles, M. (2004). Simplement, seulement, malheureusement, heureusement. Travaux de Linguistique, 49(2), 57-79.

Lamiroy, Béatrice. (2005). La grammaticalisation des adverbes autrement vs anders. In: Choi-Jonin, I., Bras, M., Dagnac, A. \& Rouquier, M. (eds.), Questions de classification en linguistique (177-195). Bern: P. Lang.

Le Bidois, R. (1952). L'Inversion du sujet dans la prose contemporaine (1900-1950). Paris: Artrey.

Marandin, J.-M. (2001). Unaccusative Inversion in French. In Y. D’Hulst, J. Rooryck, \& J. Schroten (Eds.), Going Romance 1999. Amsterdam: John Benjamins.

Marandin, J.-M. (2003). Inversion du sujet et structure de l'information dans les langues romanes. In D. Godard (Ed.), Langues romanes. Problèmes de la phrase simple (pp. 195-222). Paris: Editions du CNRS.

Molinier, C. (2012). Ainsi : deux emplois complémentaires d'un adverbe type. In J. Radimsky \& I. Mirto (Eds.), Autour de l'adverbe (pp. 120-128). Amsterdam: John Benjamins.

Molinier, C., \& Levrier, F. (2000). Grammaire des adverbes : description des formes en -ment. Genève: Librairie Droz S.A.

Riegel, M., Pellat, J.-C., \& Rioul, R. (2011). Grammaire méthodique du français. Paris: Presses Universitaires de France.

Roberts, I., \& Roussou, A. (2003). Syntactic change: a minimalist approach to grammaticalisation. Cambridge: Cambridge University Press.

Traugott, E. C. (1997). The role of the development of discourse markers in a theory of grammaticalization, 1-23. Présenté à la conférence ICHL XII à Manchester (1995). http://www.wata.cc/forums/uploaded/136_1165014660.pdf

Zenone, A. (1982). La consécution sans contradiction : donc, par conséquent, alors, ainsi, aussi (première partie). Cahiers de Linguistique Française, 4, 107-141.

\footnotetext{
${ }^{1}$ Hybertie (1996), Guimier (1997) et Flament-Boistrancourt (1999) présentent également l'emploi dit 'constatif' d'ainsi. Ce type d'ainsi établit selon ces auteurs une relation de conformité entre (l'état de choses représenté dans) l'énoncé et la situation d'énonciation. L'ajout d'ainsi souligne le fait que l'on constate quelque chose dans la situation même. Dans l'exemple (i), le locuteur constate en effet que l'interlocuteur s'est coupé les cheveux.
}

(i) Ainsi 'constatif'

Ainsi tu t'es coupé les cheveux ! (Hybertie 1996 : 52, Guimier 1997 : 85)

Cet emploi d'ainsi semble réservé à la langue parlée, et comme notre corpus n'en contient pas d'exemples, nous l'excluons de notre analyse.

${ }^{2}$ Flament-Boistrancourt (1999) et Lahousse (2011) donnent également des exemples d'ainsi de conséquence + inversion nominale (ii) :

(ii) a. C'est de sa mère même qu'elle a reçu son fiancé : ainsi se referme hermétiquement autour de Charlotte sa maison-coquillage. (Gracq, dans Lahousse 2011 : 94)

b. Mettons la réunion plutôt, ainsi pourra être abordée cette question qui vous tient à cœur. (FlamentBoistrancourt 1999 : 149) 
3 Une caractéristique qui distingue ainsi de manière et ainsi quotatif d'un côté d'ainsi adverbe de phrase (conséquence, précision, illustratif) de l'autre, est que les premiers ne peuvent jamais être suivis d'une virgule (iii).

(iii) a. * Ainsi de manière + virgule

Le 21 janvier 1995, l'ETA abattait, en plein centre de Saint-Sébastien, le dirigeant du PP au Pays basque et adjoint au maire de cette ville. * Ainsi, commençait la série macabre [de meurtres]. (exemple basé sur M550)

b. * Ainsi quotatif + virgule

"Il ne cherche plus qu'à animer le peuple contre le souverain pontife pour qu'on se révolte contre lui et ses ministres, (...).»* Ainsi, dit Machiavel d'un dominicain condamné au bûcher, ainsi beaucoup ont pensé et écrit de Raymond Léopold Bruckberger. (exemple basé sur M237)

Ainsi en tant qu'adverbe de phrase, par contre, peut précéder une virgule (iva). Il est à noter, cependant, qu'il n'est pas toujours possible d'insérer une virgule (ivb), et que la virgule est plus naturelle quand ainsi est suivi de l'ordre des mots Sujet Verbe (ive).

(iv) a. Ainsi adverbe de phrase + virgule (exemple authentique)

A l'exception des Bourses de Milan et de Madrid, toutes les places terminent la semaine dans le rouge. Ainsi, la Bourse de Singapour affiche-t-elle un recul record de 22,52\%, celle de Djakarta a perdu 16,35\%. (M195)

b. * Ainsi adverbe de phrase + virgule

Ses calembours suintent l'antisémitisme et il ne rate jamais une allusion au génocide juif pour en relativiser l'ampleur. * Ainsi, déclarait-il, le 20 novembre 1996, qu' « en France, en 1943, on ne traitait pas les juifs comme on traite aujourd'hui les gens du Front national ».

c. Ainsi + virgule + Sujet Verbe

Bien qu'il soit encore trop tôt pour dresser un bilan global d'El Niño 1997-1998, les dégâts qu'on peut lui imputer sont déjà considérables. Comme les modèles l'avaient suggéré, le phénomène a décalé vers l'est les précipitations tropicales et les cyclones. Ainsi, les zones habituellement sèches sont devenues humides, et, à l'inverse, les régions pluvieuses ont été frappées de sécheresse. (M449)

Nous remercions le lecteur anonyme qui nous a signalé l'intérêt de la virgule.

${ }^{4}$ Bien sûr, notre hypothèse devra être testée dans un corpus diachronique. De plus, il serait également intéressant d'examiner le même adverbe en d'autres langues. Les équivalents germaniques d'ainsi, 'so'/'thus' (anglais), '(al)so' (allemand) et 'zo' (néerlandais), par exemple, font preuve de la même polysémie : l'interprétation de manière, l'emploi quotatif et la conséquence pour l'anglais, et en allemand et en néerlandais il s'y ajoute également l'interprétation illustrative. Les deux exemples suivants montrent l'emploi illustratif en allemand (va) et en néerlandais (vb), et l'impossibilité d'une traduction en anglais avec 'so' ou 'thus' (vc); ces adverbes ont une interprétation consécutive :

(v) a. Néerlandais : 'zo' ('ainsi') illustratif

Officieel is de wijk Oost in 2007 bestempeld als probleemwijk, echter zijn er ook positieve kanten van Amsterdam Oost. Zo is er bijvoorbeeld een mooi buitenzwembad genaamd de Flevoparkbad dat geschikt is voor alle leeftijden. (http://1-plaatsen.plazilla.com/woon-en-leefomgeving-amsterdam)

Officiellement, le quartier Oost ('Est') a été désigné comme un quartier problématique en 2007, mais Amsterdam Oost a également ses côtés positifs. Ainsi il y a par exemple une belle piscine, la Flevoparkbad, pour tous les âges.

b. Allemand : 'so' ('ainsi') illustratif

Kaum eine Sprache hat Formen für alle Konjugationsklassen. So gibt es zum Beispiel im Chinesischen oder Japanischen weder Person noch Numerus. (http://de.wikipedia.org/wiki/Konjugation_(Grammatik)) 
Presque aucune langue n'a des formes pour toutes les classes de conjugaison. Ainsi les langues chinoise et japonaise ne marquent-elles par exemple ni la personne ni le nombre.

c. Anglais: *'so' ('ainsi') illustratif

* So / thus, there is for example a nice swimming pool.

* So / thus Chinese and Japanese for example lack person and number marking.

${ }^{5}$ Ainsi et par exemple se trouvent parfois dans la même phrase (via). Cela ne va pas à l'encontre de notre analyse d'ainsi illustratif comme exprimant par exemple, car il n'est pas exceptionnel que deux adverbes ayant le même sens apparaissent dans la même phrase (cf. des phrases contenant également et aussi, (vib)).

(vi) a. Ainsi + par exemple dans la même phrase

De l'autre côté, des établissements d'élite sont protégés et souvent surdotés en moyens. Ainsi, la répartition des postes de personnels ATOS (administratifs, techniques, ouvriers et de service), par exemple, est-elle la chose au monde la plus mal partagée (...). (M443)

b. Également + aussi dans la même phrase

Outre la chasse il y a également la pêche qui est, elle aussi, une menace pour la préservation des espèces. (www)

${ }^{6}$ Voir section 3.2 pour la distinction entre la conséquence voulue et la conséquence non intentionnelle.

${ }^{7}$ Notre analyse prend uniquement en compte les occurrences d'ainsi en tête d'une phrase avec un nœud verbal. Les constructions suivantes ont donc été exclues de notre analyse : les phrases où ainsi modifie un participe passé (viia), où ainsi introduit une phrase sans verbe (viib,c) ou une phrase enchâssée (viid), la conjonction ainsi que (viie) et des expressions figées telles qu'ainsi soit-il, ainsi en est-il de et ainsi de suite (viif).

(vii) a. Ainsi posée, la question suggère que les musées français auraient pillé leurs homologues allemands, (...). (M119)

b. Ainsi de son exposition chez Durand-Dessert. (M27)

c. Ainsi ce jeune Marocain qui vit en Turquie, illégalement, depuis deux ans et «aide » ses compatriotes. (M220)

d. Ainsi, lorsqu'il a lancé : "L'idéologie peut être mortelle »ou, encore, quand il a vanté les mérites de l'adaptabilité. (M612)

e. Ainsi qu'il l'a déclaré à l'AFP, leur décision est motivée par "l'incapacité du conseil à mettre fin aux missions (...). (M129)

f. Ainsi en est-il du cautionnement. (M52) 